\title{
Validation of a small-animal PET simulation using GAMOS: a GEANT4-based framework
}

\author{
M Cañadas, P Arce and P Rato Mendes \\ CIEMAT (Centro de Investigaciones Energéticas. Medioanbientales y Tecnológicas), \\ Madrid. Spain \\ E-mail: matio.canadas
}

\begin{abstract}
Monte Carlo-based modelling is a powerful tool to help in the design and optimization of positron emission tomography (PET) systems. The performance of these systems depends on several parameters, such as detector physical characteristics, shielding or electronics, whose effects can be studied on the basis of realistic simulated data. The aim of this paper is to validate a comprehensive study of the Raytest ClearPET small-animal PET scanner using a new Monte Carlo simulation platform which has been developed at CIEMAT (Madrid, Spain), called GAMOS (GEANT4-based Architecture for Medicine-Oriented Simulations). This toolkit, based on the GEANT4 code, was originally designed to cover multiple applications in the field of medical physics from radiotherapy to nuclear medicine, but has since been applied by some of its users in other fields of physics, such as neutron shielding, space physics, high energy physics, etc. Our simulation model includes the relevant characteristics of the ClearPET system, namely, the double layer of scintillator crystals in phoswich configuration, the rotating gantry, the presence of intrinsic radioactivity in the crystals or the storage of single events for an offline coincidence sorting. Simulated results are contrasted with experimental acquisitions including studies of spatial resolution, sensitivity, scatter fraction and count rates in accordance with the National Electrical Manufacturers Association (NEMA) NU 4-2008 protocol. Spatial resolution results showed a discrepancy between simulated and measured values equal to $8.4 \%$ (with a maximum FWHM difference over all measurement directions of $0.5 \mathrm{~mm}$ ). Sensitivity results differ less than $1 \%$ for a $250-750 \mathrm{keV}$ energy window. Simulated and measured count rates agree well within a wide range of activities, including under electronic saturation of the system (the measured peak of total coincidences, for the mouse-sized phantom, was $250.8 \mathrm{kcps}$ reached at $0.95 \mathrm{MBq} \mathrm{mL} \mathrm{mL}^{-1}$ and the simulated peak was $247.1 \mathrm{kcps}$ at $0.87 \mathrm{MBq} \mathrm{mL}^{-1}$ ). Agreement better than $3 \%$ was obtained in the scatter fraction comparison study.
\end{abstract}


We also measured and simulated a mini-Derenzo phantom obtaining images with similar quality using iterative reconstruction methods. We concluded that the overall performance of the simulation showed good agreement with the measured results and validates the GAMOS package for PET applications. Furthermore, its ease of use and flexibility recommends it as an excellent tool to optimize design features or image reconstruction techniques.

\section{Introduction}

Positron emission tomography (PET) dedicated to animal studies is an essential imaging modality for preclinical research (Hutchins et al 2008, Cherry and Gambhir 2001). Imaging small objects pushes the limits of PET technology and encourages design innovations, especially in small-animal systems that are required to fulfil both high spatial resolution and high sensitivity (Lewellen 2008, Rowland and Cherry 2008). Monte Carlo simulation is a powerful tool to study the effect of several parameters of a PET scanner, such as type of crystal and detectors, geometry and dimensions of the field of view (FOV), electronics or shielding, on its final performance. In nuclear medicine, realistic simulated data can also be used for optimizing image reconstruction methods and in the development and evaluation of image correction techniques (Zaidi 1999),

Different Monte Carlo programs have been in use in the field of nuclear imaging and internal dosimetry with many of them available as open source codes. They can be categorized into two groups: programs built on top of a general-purpose code, such as Sim-SPECT (Yanch and Dobrzeniecki 1993) based on MCNP (Briesmeister 2000), PET-EGS (Castiglioni et al 1999) based on EGS4 (Nelson et al 1985), PeneloPET (España et al 2009) based on PENELOPE (Sempau et al 1997) or GATE (Jan et al 2004) based on GEANT4 (Agostinelli et al 2003); and dedicated software packages developed for nuclear medicine imaging applications such as SIMSET (Lewellen et al 1998), PETSIM (Thompson et al 1992), EIDOLON (Zaidi ef al 1999) or PET-SORTEO (Reilhac et al 2004). Software packages based on general-purpose codes have the advantage of using validated physics and geometry transportation.

The GAMOS (GEANT4-based Architecture for Medicine-Oriented Simulations) (Arce et al 2008) toolkit is based on the GEANT4 code and covers several medical applications, from radiotherapy to nuclear medicine. As GATE, the other currently available GEANT4based framework for nuclear medicine, the first objective of GAMOS is to facilitate the use of GEANT4 without requiring the knowledge of $\mathrm{C}++$ programming language, providing instead a set of user commands. Nevertheless, the main difference of GAMOS with respect to other programs lies in its flexibility, which has also been a main objective since its first design. To achieve this, GAMOS scripting-language ofters a considerable amount of possibilities for the implementation of a new model, the analysis of the underlying physical process and the generation of output data. The input parameters are open to any isotope, material or physics lists that can be found in the GEANT4 code. With regard to the output, apart from the histograms and files necessary to emulate the performance of an imaging system, the user can gather detailed information about the simulation: histograms and text or binary files can be filled with many types of data, which can be sorted with several classifiers, such as histograms per primary particle, per volume or per radius interval. Another source of flexibility is the possibility of adding new functionalities; this is achieved thanks to the plug-in concept: a user can write a new component or take it from the GEANT4 examples, transform it into a plug-in 
and select it in the initial script, mixing it with the GAMOS standard components. Step-bystep examples of each plug-in type have been included in the on-line available documentation (GAMOS 2010).

The accuracy of a code is determined by its capability to generate data identical to those that are obtained on real systems. For this reason, simulation of a PET scanner must be validated by comparing its performance assessment against measured results. The comparison must be carried out under the same conditions in issues such as the image reconstruction protocol or the radioisotope used, which may significantly affect the results (Weber and Baner 2004). We agree with Buvat et al (2005) that a validation protocol should be based on standards of the National Electrical Manufacturers Association (NEMA) for PET systems, which are widely accepted and for which results have been already published for a number of scanners. These protocols evaluate the performance of a PET system in terms of spatial resolution, sensitivity, count rates, scatter fraction and image quality. We evaluate the GAMOS model of the ClearPET (Raytest GmbH, Mannheim, Germany) (Mosset et al 2004) small-animal scanner by comparing its simulated performance against measured acquisitions following a protocol based on the NEMA NU 4-2008 standard (NEMA 2008). The main interest of this work is to show the ability of GAMOS to generate realistic simulated data of a state-of-the-art preclinical PET scanner such as the ClearPET, composed of a full ring of detectors based on lutetium-yttrium orthosilicate (LYSO) and lutetium-yttrium aluminum perovskite (LuYAP) phoswich scintillator crystals coupled to position-sensitive photomultiplier tubes (PMT).

\section{Materials and methods}

\subsection{The ClearPET scanner}

All the measurements have been carried out on the ClearPET scanner located at CIEMAT (Centro de Investigaciones Energéticas, Medioambientales y Tecnológicas, Madrid, Spain). Table 1 shows a summary of the geometrical and physical characteristics of the system.

The detector modules in the ClearPET can change the inner diameter from 13.5 to $22 \mathrm{~cm}$ for mouse/rat or primate imaging; however, this work focuses only on the small-diameter configuration. The data acquisition system consists of 20 detector cassettes, each of them comprising four PMTs, and electronics for trigger, shaping, data-digitization and transmission. These cassettes transfer their data via fibre optics to five preprocessing PCs (four cassettes per PC) which provide list-mode files with the information of each single photon detected. From there, a giga-bit ethernet connection leads the preprocessed data to a master PC, which also controls the scan process and hosts the interface for the user (Streun et al 2006). Coincidence sorting is performed in the master computer after acquisition, and events are written in listmode format files that can be sorted into sinograms and reconstructed by the STIR (Software for Tomographic Image Reconstruction) package (Thielemans et al 2006).

\subsection{GAMOS simulation model}

In GAMOS, any PET geometry can be defined with a simple text format (GAMOS 2010). Apart from this, there is a utility to simulate simple PET detectors by just providing a few parameters, namely number of crystals per block, number of blocks per ring, number of rings, radial, transaxial and axial crystal size, and detector ring diameter. Since the ClearPET system has an axial shift of $9.2 \mathrm{~mm}$ between each two adjacent detector modules, a specific geometry description was implemented using the text format. The geometry definition considers a full ring with 20 detector cassettes (figure 1), each of them composed of four matrices of crystals 
Table 1. Characteristics of the ClearPET.

\begin{tabular}{ll}
\hline Detector & \\
Detector ring diameter (mm) & $135 / 220^{\mathrm{a}}$ \\
Number of detector modules & 20 \\
Number of PMTs & 80 \\
PMT type & Position-sensitive. Hamamatsu R7600-M64 \\
Layers of crystals, radial direction & 2 \\
Crystal size (mm $)$ & $2 \times 2 \times 10^{\mathrm{b}}$ \\
Crystal pitch (mm) & 2.3 \\
Crystal material & LYSO/LuYAP \\
Systent & \\
Maximum transaxial field of view (FOV) (mm) & $94 / 144^{\mathrm{a}}$ \\
Axial FOV (mm) & 110 \\
Rotating gantry & Yes. 1 revolution per minute \\
Coincidence window (ns) & 12 \\
Energy windows (keV) & $100-750$ \\
& $250-750$ \\
& $400-750$ \\
Image pixel size (mm) & $1.15 / 0.57 / 0.38$ \\
Slice thickness (mm) & 1.15 \\
Reconstruction methods & $2 \mathrm{D} / 3 \mathrm{D} \mathrm{FBP}$ \\
& $2 \mathrm{D} / 3 \mathrm{D}$ OSEM
\end{tabular}

${ }^{3}$ Two adjustable detector diameters for rodent whole body or primate brain studies.

b Two radial layers of $10 \mathrm{~mm}$.

${ }^{\mathrm{c}}$ Front layer: LYSO, back layer: LuYAP.

${ }^{\mathrm{d}}$ Filtered back projection.

${ }^{e}$ Ordered subsets expectation maximization (Hudson and Larkin 2004).

(an $8 \times 8$ LYSO/LuYAP dual-layer block, dimensions shown in table 1), and other passive materials such as gantry, the plastic bed and aluminium covers in order to account for radiation dispersion and backscattering which may increase the coincidence background and degrade the image quality. GAMOS has also the possibility of simulating any kind of translation or rotation of the solids included in the geometry. As ClearPET has a gantry with a continuous rotation movement ( 1 revolution per minute), we decided to use $2^{\circ}$ steps to simulate this rotation.

As an example, we show the part of the text file that defines the $8 \times 8$ matrix of phoswich crystals (they are placed inside the solid named module). The label : $P$ defines the parameters to be called through the file. The label :VOLU defines a GEANT4 logical volume and :PLACE is used for the placement of a volume (:PLACE_PARAM is used for a parameterized placement, linear, square or circle).

//The number of radial and tangential crystals are defined as parameters:

:P NCrystal_ax 8

:P NCrystal_tran 8

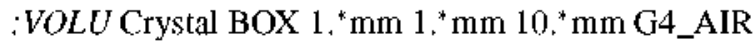

// Using a predefined material from GEANT4 database 

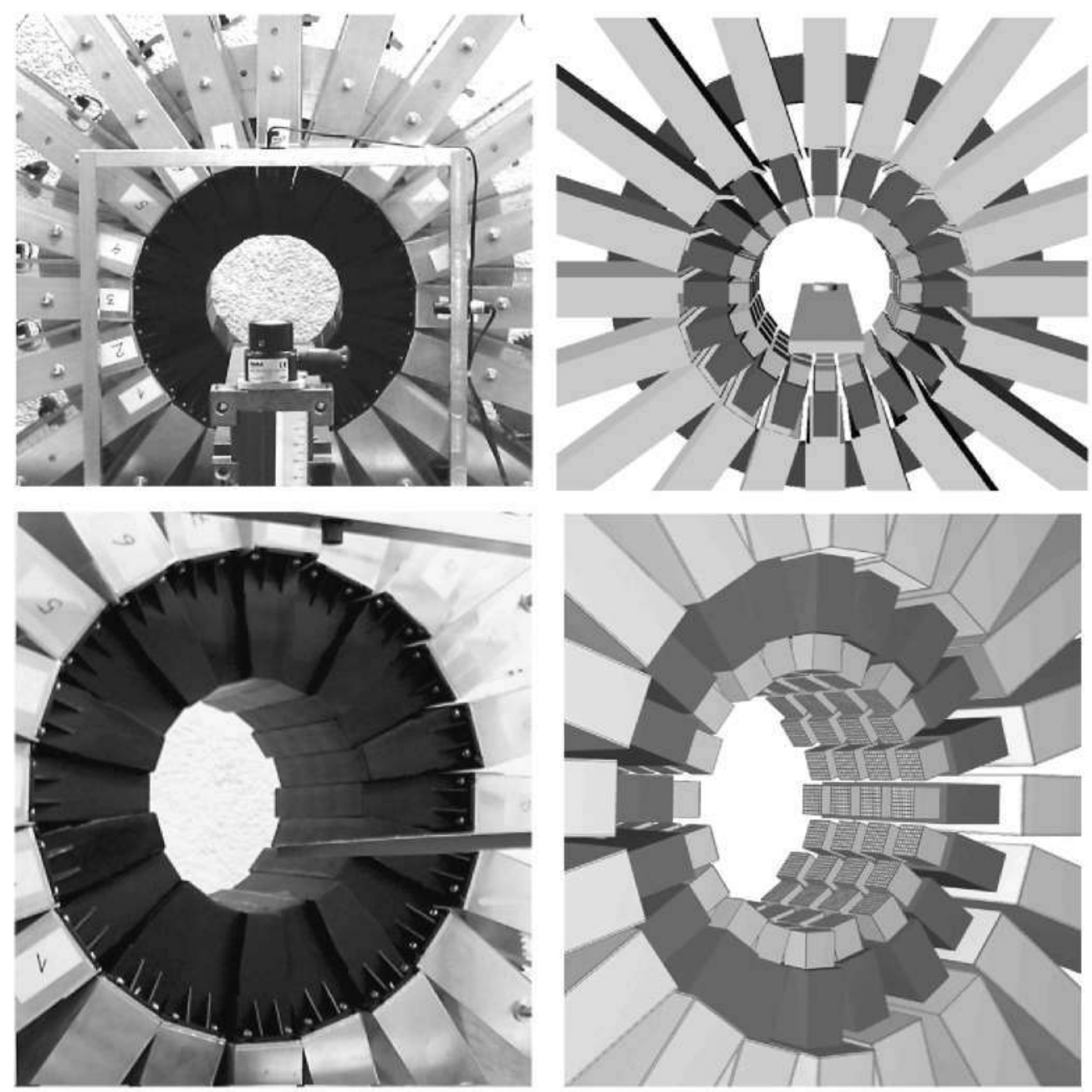

Figure 1. Full ring of detector cassettes of the ClearPET (left) and its GAMOS model (right), including crystals and passive materials such as gantry, covers and bed. Detailed view (bottom) showing the axial offset between each two adjacent detectors.

:PLACE_PARAM Crystal 0 Module SQUARE_XY RM0 \$NCrystal_ax \$NCrystal_tran $2.3^{*} \mathrm{~mm} 2.3^{*} \mathrm{~mm}-1.15^{*}$ (\$NCrystal_ax-1) $-1.15^{*}$ (\$NCrystal_ax-1)

// LYSO and LuYAP radial layers are placed inside the crystals defined above. :VOLU Layer0 BOX 1. ${ }^{*} \mathrm{~mm} 1 .{ }^{*} \mathrm{~mm}$ 5. ${ }^{*} \mathrm{~mm}$ LYSO // Using a predefined material from

:PLACE Layer0 0 Crystal R000 00 0 -5.* mm GAMOS database

:VOLU Layer1 BOX 1. ${ }^{*} \mathrm{~mm} 1 .{ }^{*} \mathrm{~mm}$ 5. ${ }^{*} \mathrm{~mm}$ LuYAP

:PLACE Layer1 0 Crystal R000 00 5. ${ }^{*} \mathrm{~mm}$

Two positron emitters were considered: ${ }^{18} \mathrm{~F}$ for count rate studies, and ${ }^{22} \mathrm{Na}$ for spatial resolution, sensitivity and mini-Derenzo phantom image quality studies. Positron emission energy is generated following the respective continuous distribution of the beta-decay process, 
obtained from the LBNL/LUND table of radioactive isotopes (Firestone and Ekstrom 1999). All the electromagnetic processes were simulated using the GEANT4 low-energy extension of electromagnetic interactions. An important characteristic of the ClearPET is its natural radioactivity in the crystals due to the presence of naturally occurring ${ }^{176} \mathrm{Lu}$ (both in the LYSO and LuYAP layer). This isotope emits $\beta^{-}$particles with an average energy of $420 \mathrm{keV}$, together with $\gamma$-photons of 307,202 and $88 \mathrm{keV}$, all of which increase the single count rate of the detectors and may create events that can be recognized as true coincidences (Yamamoto et al 2005). This has been simulated by adding a second source with a total activity of $178 \mathrm{kBq}$ in the crystals, at $550 \mathrm{~Bq} \mathrm{~cm}^{-3}$ in LYSO and $150 \mathrm{~Bq} \mathrm{~cm}^{-3}$ in LuYAP. These numbers were selected to match the ClearPET intrinsic counting rate and are in accordance with those published (Thiel et al 2006, Baberdin et al 2008).

With regard to the detection process, in GAMOS the user may select one of the predefined sensitive detectors available, or alternatively use a custom one to produce the signals (hits) and eventually digitize and reconstruct them. For these studies, an event is stored only if an amount of energy within the energy window is deposited in one or several neighbour pixels of the same layer, in which case the interaction point is assigned to the pixel with maximum energy deposition. We have assumed perfect layer discrimination. The energy resolution of the detectors is simulated by using the GAMOS functionality to smear the energy deposition. The smearing is done following Gaussian distributions corresponding to energy resolutions of $23.2 \%$ (LYSO) and $24.3 \%$ (LuYAP) full-width at half-maximum (FWHM) at $511 \mathrm{keV}$ (Ziemons et al 2003). We have selected a measuring time of $400 \mathrm{~ns}$ and a paralyzable dead time of $850 \mathrm{~ns}$, at the level of the cassettes. The maximum single rate for the whole system is limited to 6.8 Mcounts $\mathrm{s}^{-1}$, in order to simulate the saturation of the data transfer from cassettes to the master PC.

We have also developed a module to simulate in detail the front-end electronics of the ClearPET and replicate the layer discrimination inside the block of crystals. ClearPET uses a depth of interaction (DOI) assessment based on the assumptions that the front-end amplifiers are linear and the shapes of the filtered pulses depend only on the type of crystal where the interaction occurred (LYSO or LuYAP) and the total energy deposited there; we have emulated this process by obtaining the pulse shape from fits of continuous functions to published ClearPET ADC data (Streun et al 2003). Although this module can be used to study accurately the DOI process (taking into account the occurrence of pile-up events), the presented results were obtained using the standard sensitive detectors of GAMOS, which require less CPU time and showed no significant differences on the overall performance of the system. For the count rate studies, the limitation on the single event rate is the main factor to reproduce the scanner behaviour and we show the effect of its inclusion in the results section.

Coincidence sorting was done using a 12 ns window, and images were reconstructed after acquisition following the same data flow as the scanner. A new feature to write simulated single events using the ClearPET list-mode format was developed in order to compare results from the same reconstruction methods; furthermore, as ClearPET uses the open-source STIR package, this functionality has been transformed into a GAMOS plug-in to make it available for other GAMOS users interested on PET image reconstruction. STIR is an image reconstruction toolkit suitable for multiple PET data formats (Thielemans et al 2006), including those of several clinical scanners.

\subsection{Performance evaluation}

The methodology used to evaluate the performance of the simulation includes studies of spatial resolution, sensitivity, scatter fraction and count rate measurements (focused on mouse 
imaging) following the recommendations of the NEMA NU 4-2008 standard (NEMA 2008), created to evaluate small-animal PET systems (Bao et al 2009, Wietholt et al 2008, Luo et al 2010). In addition, we show an acquisition without radioactive sources in the FOV, to evaluate the intrinsic radiation from ${ }^{176} \mathrm{Lu}$, and an image of a Derenzo phantom reconstructed with the iterative methods available in the ClearPET.

2.3.1. Spatial resolution. A $0.25 \mathrm{~mm}$ diameter $0.8 \mathrm{MBq}{ }^{22} \mathrm{Na}$ point source was scanned at equivalent positions in the real system and in the simulated scenario. The measurements were taken at the central slice of the axial FOV, at the following radial distances from the geometrical centre: $0,5,10,15,20$ and $25 \mathrm{~mm}$. Resolution at the same transaxial points was also evaluated at $\frac{1}{4}$ axial FOV. Although acquisition at $0 \mathrm{~mm}$ is not included in the NEMA document, results at this location have been presented in previous characterizations of the system (Canadas et al 2008 , Roldan et al 2007). More than $10^{5}$ prompt counts were acquired per measurement, and analytic image reconstruction algorithms with no-smoothing filters were applied: a 3D filtered back projection algorithm (the FBP3DRP algorithm from the STIR package) with an image pixel size of $0.38 \mathrm{~mm} \times 0.38 \mathrm{~mm}$ and a slice thickness of $1.15 \mathrm{~mm}$. The reported values characterize the width of the reconstructed image point spread functions (PSF), defining the width as its FWHM amplitude and the full width at tenth-maximum amplitude (FWTM). The response function is formed by summing all one-dimensional profiles that are parallel to the direction of measurement (radial, tangential or axial) and within two times the FWHM of the orthogonal directions. The fitting method used to assess each FWHM (and FWTM) fulfils the indications of the NEMA NU 4-2008 protocol.

2.3.2. Sensitivity. Sensitivity is expressed as the rate in counts per second (cps) at which true coincidence events are detected for a given source intensity and branching ratio; absolute sensitivity is the fraction of positron annihilation events detected as true coincidence events. For sensitivity studies the same ${ }^{22} \mathrm{Na}$ point source previously described was scanned in small position increments along the entire axial FOV.

Following the NEMA NU-4 protocol, we subtracted the background counts due to the intrinsic radioactivity, both in measurement and simulation. At every axial position, $i$, sensitivity $\left(S_{j}\right)$ is then calculated as shown in

$$
S_{i}=\frac{R_{i}-R_{B . i}}{A_{\text {cal }}}
$$

where $A_{\text {cal }}(\mathrm{MBq})$ is the activity of the source, $R_{i}$ is the rate $(\mathrm{kcps})$ of total counts collected in slice $i$, after applying a single-slice rebinning (SSRB) algorithm (Daube-Witherspoon and Muehllehner 1987), and $R_{B, i}$ is the background total event rate acquired without sources in the FOV. The parameters $S_{\text {tot }}$ and $S_{\text {tot }}$ represent the total and total mouse axial length sensitivities and are computed as the average of $S_{i}$ over the whole axial FOV and over the central $7 \mathrm{~cm}$, respectively. Their values are also reported as the absolute sensitivities $\left(S_{A, t o t}, S_{A \text { Atot }}\right)$. Since the branching ratio of ${ }^{22} \mathrm{Na}$ is 0.9060 , the absolute sensitivity $S_{A}$ is given by

$$
S_{A}=\frac{S_{i}}{9.060}
$$

where $S_{A}$ is expressed as a percentage and $S_{i}$ as kcps $\mathrm{MBq}^{-1}$.

We also present the sensitivity profiles by plotting the absolute sensitivity for each slice. As these variables are strongly dependent on the energy window considered, the NEMA values are presented using the energy window recommended for routine studies $(250-750 \mathrm{keV})$.

In addition to these results, we have measured and simulated a sensitivity profile for the widest window achievable on the system $(100-750 \mathrm{keV})$; the widest window is recommended 
by the manufacturer for low-dose studies. We also compare the sinograms obtained at the maximum sensitivity point of the scanner, which is not reached in the centre of the FOV due to the axially shifted detectors.

2.3.3. Scatter fraction and count rates. For scatter fraction and count rate measurements we used the mouse-sized phantom recommended by the NEMA NU-4 2008 protocol. This phantom is a solid cylinder composed of high-density polyethylene (density $0.95 \mathrm{~g} \mathrm{~cm}^{-3}$ ) 70 $\mathrm{mm}$ long and $25 \mathrm{~mm}$ in diameter. A cylindrical hole $(3.2 \mathrm{~mm}$ in diameter) is drilled parallel to the central axis at a radial distance of $10 \mathrm{~mm}$. A line source made of flexible tubing and filled to $60 \mathrm{~mm}$ with a known activity concentration of ${ }^{18} \mathrm{~F}$ is inserted into the hole.

Count rate measurements show the effects of system dead-time at different levels of source activity. We present results on the total coincidences acquired for the $250-750 \mathrm{keV}$ energy window and the noise equivalent counting (NEC) rate, which describes the equivalent coincidence counting rate that would have the same noise properties as the net true counting rate, corrected for random and scattered events (Strother et al 1990), defined as

$$
R_{\mathrm{NEC}}=\frac{R_{t}^{2}}{R_{\mathrm{TOT}}}
$$

where $R_{\text {TOT }}$ represents the rate of total coincidences and $R_{f}$ the rate of true coincidences. For each acquisition, a SSRB algorithm is carried out in order to obtain a projection profile by summing all projections of the sinogram of each axial slice. Then, $R_{f}$ is calculated as the sum of all counts inside a $14 \mathrm{~mm}$ strip, centred in the peak, minus the scattered and random counts. The scattered and random counts under the peak are estimated by a linear interpolation between the left and right border of the $14 \mathrm{~mm}$ strip (as described in the NEMA document).

The system scatter fraction (SF) represents the percentage of scattered events for a given phantom. We present the simulated and measured scatter fractions for the mouse-sized phantom; as ClearPET crystals have intrinsic radioactivity, its effect was taken into account by subtracting the profile of intrinsic radiation rate, $R_{\text {int }}$.

These studies start with high activity in the phantom, exceeding the expected maximum count rate. The measurements were performed using the energy window recommended for routine studies $(250-750 \mathrm{keV})$. The phantom was centred in the FOV and the acquisition protocol was selected as follows: frames of $300 \mathrm{~s}$ starting each $1200 \mathrm{~s}$. The initial activity concentration was $1.25 \mathrm{MBq} \mathrm{mL}^{-1}$ (43 MBq total activity of ${ }^{18} \mathrm{~F}$ ). Similar levels of activities and total events were considered for the simulated acquisition. The SF was computed from the low activity acquisitions, those with a total activity in the phantom below $5 \mathrm{MBq}$.

2.3.4. Background of intrinsic radioactivity. Count rate studies require the measurement of the intrinsic radioactivity rate, $R_{\text {int }}$. We evaluated $R_{\text {int }}$ due to ${ }^{176} \mathrm{Lu}$ for the $250-750 \mathrm{keV}$ energy window, both in the real measurements as well as in the simulation analysis. Data were collected from a 5 min acquisition $\left(\sim 10^{5}\right.$ counts). In addition, images of the generated sinograms (after SSRB) are presented to show the effect of the gantry rotation.

2.3.5. Mini-Derenzo phantom images. A mini-Derenzo ${ }^{22} \mathrm{Na}$ sealed phantom was acquired and reconstructed with real and simulated data. The acquisition of a Derenzo image is a common way to illustrate the spatial resolution of a scanner and evaluate its overall image quality. The phantom presented a total activity of $0.76 \mathrm{MBq}$. It is composed of 20 rods measuring $1.2 \mathrm{~mm}$ in diameter, 14 rods measuring $1.5 \mathrm{~mm}, 9$ rods measuring $2.0 \mathrm{~mm}, 6$ rods measuring $2.5 \mathrm{~mm}$ and 3 rods measuring $3.0 \mathrm{~mm}$. The rods of equal diameter are distant from 


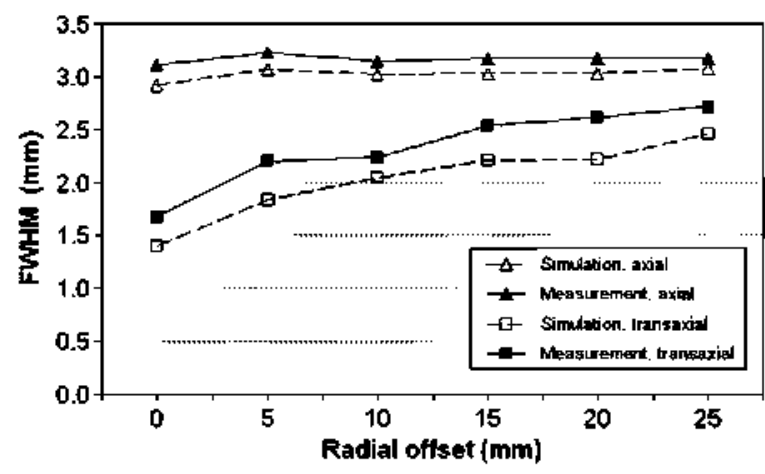

Figure 2. Sinjulated and measured NEMA spatial resolution (FWHM) along axial and transaxial (average radial and tangential) directions as a function of the tadial distance to the centre. Source positioned at the axial centre of the FOV.

each other by twice its diameter, centre to centre. The external dimensions of the phantom are $43 \mathrm{~mm}$ in length by $40 \mathrm{~mm}$ in diameter.

The number of total coincidences was limited to only 3 millions both in the experimental acquisition and in the simulation. Image reconstruction was performed using the 3D-OSEM method implemented on the ClearPET, the OSMAPOSL algorithm from STIR package (Thielemans 2006), applying two iterations and ten subsets with no attenuation or scatter corrections.

\section{Results}

\subsection{Spatial resolution}

The average one-dimensional spatial resolution (FWHM) over the three axes measured at the centre of the FOV was $2.1 \mathrm{~mm}$ (FWTM: $4.7 \mathrm{~mm}$ ), whereas the simulated value was $1.9 \mathrm{~mm}$ (FWTM: $4.0 \mathrm{~mm}$ ). Figure 2 presents the spatial resolution obtained at the axial centre of FOV. For transaxial (radial and tangential) directions we found a maximum discrepancy of $0.5 \mathrm{~mm}$, and $0.2 \mathrm{~mm}$ for the axial direction.

The average discrepancy between simulated and measured results was $8.4 \%$ for FWHM and $8.5 \%$ for FWTM. ClearPET has a small degradation $(\sim 1 \mathrm{~mm})$ of the transaxial spatial resolution along the transverse FOV that can be observed in the simulation. The systematic difference of $-0.3 \mathrm{~mm}$ on the transaxial resolution observed in the simulated results can be due to misalignments of the real detectors, which are not present in the simulated geometry. Alignment of the detector modules is critical for rotating gantry systems, where any single displacement can introduce a systematic error in the image. The overall trend of spatial resolution variation with the radial offset is similar for both simulated and measured data.

Tables 2 and 3 show the results of the spatial resolution obtained on both cases for the NEMA measuring points.

\subsection{Sensitivity}

Total length $\left(S_{\text {tot }}\right)$ and mouse length $\left(\mathrm{SM}_{\mathrm{tot}}\right)$ sensitivity values are shown in table 4 for the energy windows recommended for routine studies $(250-750 \mathrm{keV})$. Both $S_{\text {tot }}$ and $\mathrm{SM}_{\text {tot }}$ show excellent agreement between measured and simulated results (maximum discrepancy of $0.7 \%$ ). 
Table 2. Simulated spatial resolution for 5, 10. 15.20 and $25 \mathrm{~mm}$ radial offset. FWHM (FWTM) in mm.

\begin{tabular}{lccccc}
\hline & $5 \mathrm{~mm}$ & $10 \mathrm{~mm}$ & $15 \mathrm{~mm}$ & $20 \mathrm{~mm}$ & $25 \mathrm{~mm}$ \\
\hline At axial centre & & & & & \\
Radial & $1.8(3.3)$ & $2.0(3.2)$ & $2.2(3.2)$ & $2.2(3.9)$ & $2.5(4.7)$ \\
Tangential & $1.9(4.3)$ & $2.1(5.5)$ & $2.2(5.8)$ & $2.2(4.8)$ & $2.4(5.5)$ \\
Axial & $3.1(5.7)$ & $3.0(5.6)$ & $3.0(5.6)$ & $3.0(5.6)$ & $3.1(5.7)$ \\
At 1/4 axial FOV & & & & & \\
Radial & $1.8(3.8)$ & $2.2(4.0)$ & $2.6(4.4)$ & $2.4(4.2)$ & $2.4(4.1)$ \\
Tangential & $1.9(4.0)$ & $1.8(4.1)$ & $2.4(5.3)$ & $2.3(5.2)$ & $2.5(6.9)$ \\
Axial & $3.1(5.5)$ & $3.0(5.5)$ & $3.0(5.5)$ & $3.0(5.5)$ & $3.0(5.5)$ \\
\hline
\end{tabular}

Table 3. Measured spatial resolution for 5, 10. 15.20 and $25 \mathrm{~mm}$ radial offset. FWHM (FWTM) in mm.

\begin{tabular}{lccccc}
\hline & $5 \mathrm{~mm}$ & $10 \mathrm{~mm}$ & $15 \mathrm{~mm}$ & $20 \mathrm{~mm}$ & $25 \mathrm{~mm}$ \\
\hline At axial centre & & & & & \\
Radial & $2.1(4.5)$ & $1.8(3.9)$ & $2.3(4.2)$ & $2.7(4.8)$ & $2.6(4.7)$ \\
Tangential & $2.3(4.5)$ & $2.6(6.6)$ & $2.7(5.9)$ & $2.5(5.0)$ & $2.8(6.9)$ \\
Axial & $3.2(6.0)$ & $3.2(5.8)$ & $3.2(5.9)$ & $3.2(5.9)$ & $3.2(5.8)$ \\
At 1/4 axial FOV & & & & & \\
Radial & $2.3(4.7)$ & $2.0(4.2)$ & $2.2(4.2)$ & $2.8(4.8)$ & $2.6(4.7)$ \\
Tangential & $2.4(4.6)$ & $2.6(5.7)$ & $2.7(5.4)$ & $2.6(5.1)$ & $2.8(6.9)$ \\
Axial & $3.2(5.9)$ & $3.2(5.9)$ & $3.2(5.8)$ & $3.2(5.8)$ & $3.2(5.8)$ \\
\hline
\end{tabular}

Table 4. Simulated and measured sensitivity tesults for the $250-750 \mathrm{keV}$ energy window.

\begin{tabular}{lll}
\hline & Simulation & Measurement \\
\hline$S_{\mathrm{A}, \mathrm{tot}}(\%)$ & 1.34 & 1.33 \\
$\mathrm{SM}_{A, \mathrm{tot}}(\%)$ & 1.71 & 1.72 \\
$S_{\mathrm{tot}}\left(\mathrm{keps} \mathrm{MBq}^{-1}\right)$ & 12.18 & 12.06 \\
$\mathrm{SM}_{\mathrm{tot}}\left(\mathrm{kcps} \mathrm{MBq}^{-1}\right)$ & 15.53 & 15.64 \\
\hline
\end{tabular}

Figure 3 plots the NEMA absolute sensitivity profile as a function of the axial distance to the central FOV. An important characteristic of the ClearPET is the $9.2 \mathrm{~mm}$ axial displacement between each two adjacent detectors; it produces a non-homogeneous sensitivity profile with a characteristic shape that the simulated profile also reproduces. This is the cause of the absolute sensitivity peaks, which both in simulation and measurement are reached at $\pm 5 \mathrm{~mm}$ from the axial centre of the FOV. At this central region, the simulated sensitivity is $\sim 5 \%$ lower than the measured results for the widest energy window, a discrepancy which is not observed for the $250-750 \mathrm{keV}$ energy window, and which can be due to the lower light outputs of LuYAP and LYSO at low energies, in particular for LYSO which has a light yield in the energy range from 100 to $150 \mathrm{keV}$ as low as $80 \%$ of its value at $511 \mathrm{keV}$ (Kuntner et al 2002, Pidol et al 2004). This effect has not been modelled in the simulation, so that an event in which an incident gamma suffers a Compton interaction with $\sim 100 \mathrm{keV}$ deposition on a LYSO crystal pixel, followed by photoelectric absorption of the scattered gamma on a non-neighbour crystal, will be considered as a good single event in the real detector (there will only be one pixel firing, the 


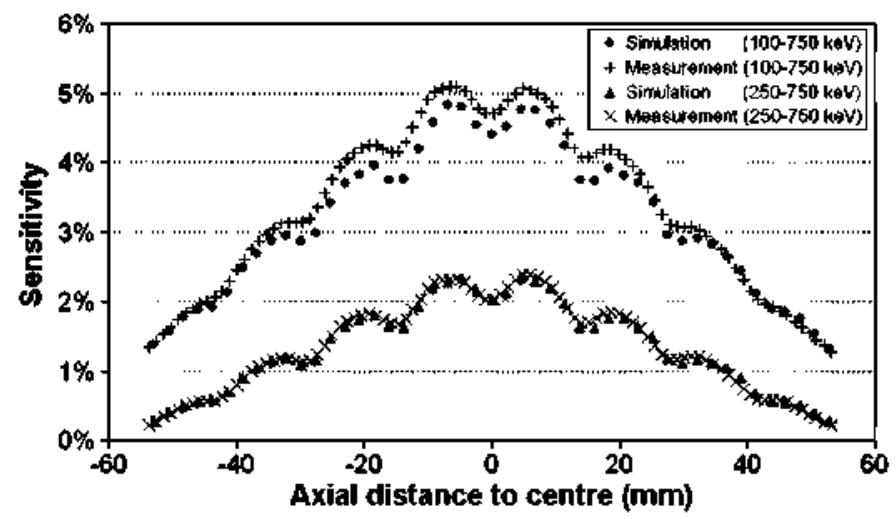

Figure 3. Sinulated and measured absolute sensitivity protiles for different energy windows.

(a)

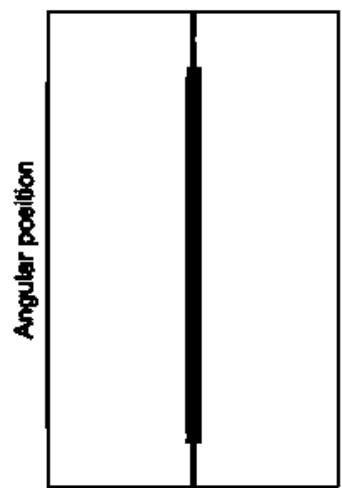

Radial position (b)

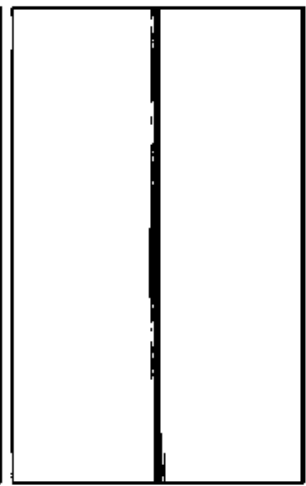

Radial position (c)

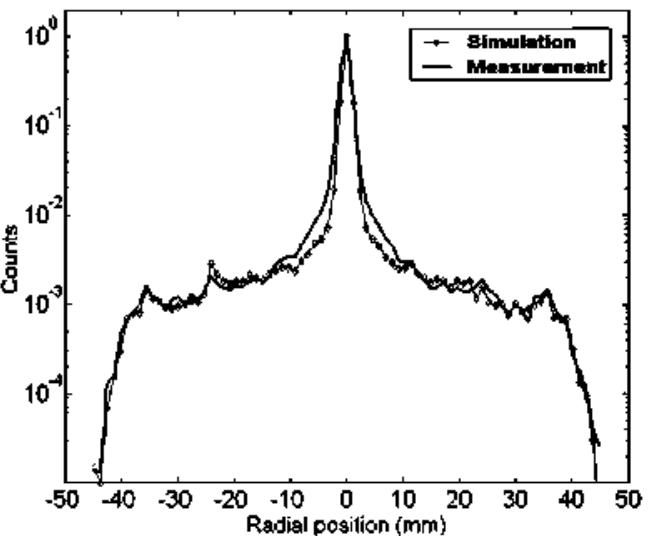

Figure 4. Simulated (a) and measured (b) sinogram for the point source positioned at $+5 \mathrm{~mm}$ from the axial centre of the FOV. Sum projection (c) of the sinograms, counts normalized to the maximum value. Data collected using the widest energy window $(100-750 \mathrm{keV})$.

low energy hit will not result in a signal above threshold due to the lower light yield), while in the simulation the same event will be discarded (there will be two non-neighbouring pixels firing in the same module). We have found that these type of events account for about $6 \%$ of the total coincidences in the centre of the FOV for data simulated using the widest energy window, a value which is in good agreement with the observed discrepancies.

Simulated and measured sinograms ( $81 \times 80$ pixels, radial and angular positions) at the maximum sensitivity point ( $\pm 5 \mathrm{~mm}$ axial offset) are shown in figure 4 . They have been formed after SSRB, using all collected counts for the widest energy window (100-750 keV). Figure 4(c) plots the profile obtained after summing all angular projections. Note that this profile includes the background produced by the natural radioactivity in the crystals.

\subsection{Scatter fraction and count rates}

The simulated SF was $31.9 \%$ and we measured a value of $31.0 \%$ for the mouse-sized phantom and $250-750 \mathrm{keV}$ energy window. Simulated total and NEC peak rate were $250.8 \mathrm{kcps}$ at 

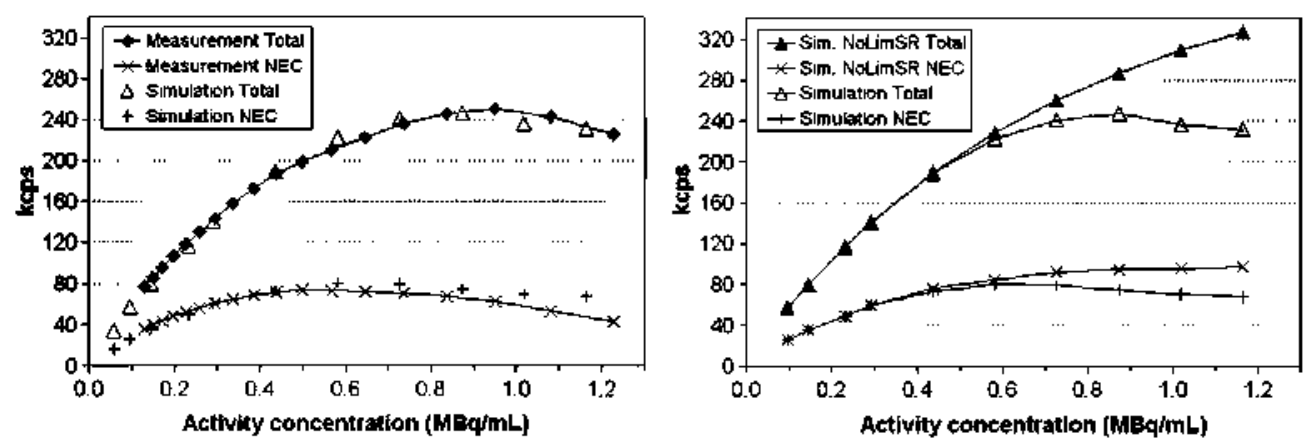

Figure 5. Total coincidences and NEC count rate as a function of the activity in the mouse-sized phanton for GAMOS simulation and experinental measurements (left). On the right. simulation tesults with and withont limitation of the maximum single rate (NoLimSR).

$0.95 \mathrm{MBq} \mathrm{mL} \mathrm{m}^{-1}$ and $73.4 \mathrm{kcps}$ at $0.50 \mathrm{MBq} \mathrm{mL}^{-1}$, respectively; the measured results were 247.1 at $0.87 \mathrm{MBq}^{-1}$ and $80.1 \mathrm{kcps}$ at $0.58 \mathrm{MBq} \mathrm{mL}^{-1}$.

Figure 5 (left) shows $R_{\mathrm{TOT}}$ and $R_{\mathrm{NEC}}$ at different activity concentrations in the mousesized phantom. The simulated and measured results agree well for a wide range of activities including under electronic saturation of the system. A discrepancy below $5.5 \%$ is observed for $R_{\text {Tor }}$ at activities smaller than $1.2 \mathrm{MBq} \mathrm{mL}^{-1}$ ( $40 \mathrm{MBq}$ of total activity). Simulated and measured NEC curves are within $8.5 \%$ at activities smaller than $0.6 \mathrm{MBq} \mathrm{mL}^{-1}$, with worse agreement found after reaching the peak.

At activities higher than $0.5 \mathrm{MBq} \mathrm{mL}^{-1}$, the modelling of the electronics and data transfer is necessary to reproduce the measured curves. Specifically, for the ClearPET, the limitation on the single data transfer is critical, as shown in figure 5 (right).

\subsection{Background of intrinsic radioactivity}

The ClearPET has an intrinsic true event rate of $560 \mathrm{cps}$ for the $250-750 \mathrm{keV}$ energy window. We added a total ${ }^{176} \mathrm{Lu}$ activity of $178 \mathrm{kBq}$ in order to match the measured intrinsic events rate and perform the count rate analysis with the same background events as the real system. Figure 6 shows the simulated and measured sinogram in the central axial slice due to the intrinsic radiation ( $\sim 10^{5}$ total counts). Sinograms were formed by applying a SSRB algorithm to the $3 \mathrm{D}$ data. We also show the sinogram obtained without rotation of the gantry (figure $6(\mathrm{c})$ ) to illustrate the importance of its inclusion in the simulation. In this case, the gaps between detectors are clearly observed.

The measured sinogram (figure 6(a)) shows a lower homogeneity than the simulated cases, which can be explained from the different behaviour between detectors, not modelled in the simulation. As these acquisitions were performed with the mouse-sized phantom (a centred cylinder without activity), higher attenuation of the radiation is observed in the centre of the FOV than in the edges. Most of the coincidences due to ${ }^{176} \mathrm{Lu}$ are produced when a beta particle is generated in one detector, and the escaped gamma photon is detected by another detector (Yamamoto et al 2005). These events explain the seven vertical strips with higher number of counts (black pixels) observed in figure 6 ; they show the centre-to-centre lines of response between each pair of detectors in coincidence. For the standard FOV configuration in the ClearPET, every cassette of detectors is in coincidence with its seven opposite cassettes. 
(a)

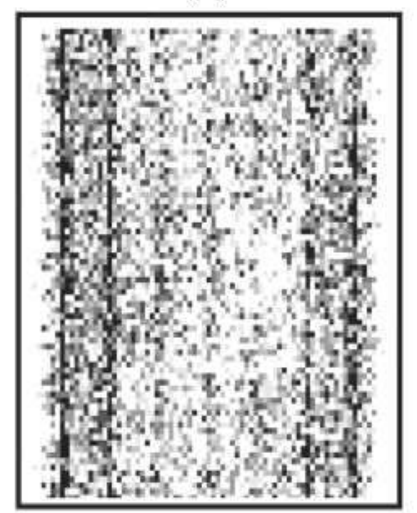

(b)

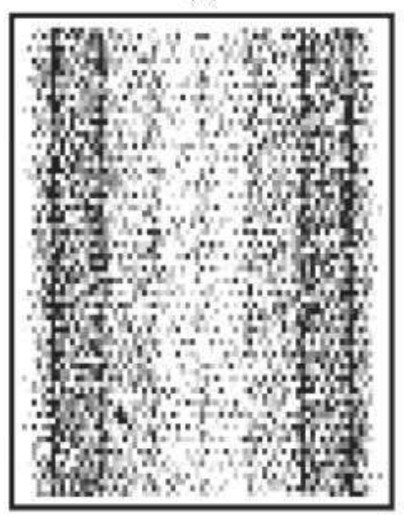

(c)

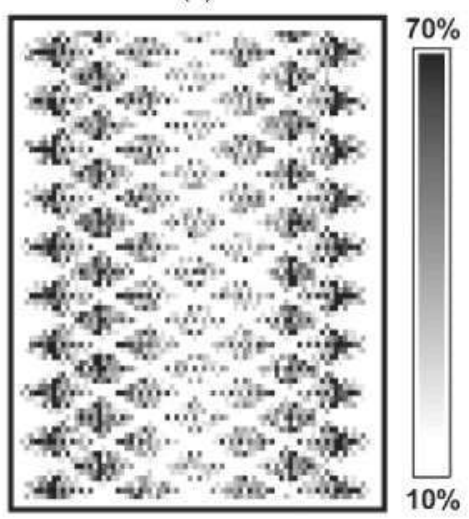

Figure 6. Sinogram at the central slice, $81 \times 80$ pixels (radial position, angular position), produced by the intrinsic radioactivity in the crystals. Acquisition using the mouse-sized phantom without activity and centred in the FOV. Measured data (a), simulation with gantry rotation using $2^{\circ}$ steps (b), simulation without gantry rotation (c).

\subsection{Mini-Derenzo phantom image}

The mini-Derenzo images are shown in figure 7. They were reconstructed using a 3D-OSEM algorithm with the same number of coincidences ( 3 million). Both images present similar image quality and good agreement between the activity profiles along the bigger rods, as can be seen for the $2.5 \mathrm{~mm}$ rods.

In both cases it is not possible to resolve the smaller rods. Nevertheless, it is important to remark that better images can be obtained in the ClearPET with longer acquisitions (Canadas et al 2008); for these studies we were only interested on comparing the acquired image against the simulation under the same number of coincidences. A 2 min acquisition was carried out on the system to collect 3 million coincidences from the mini-Derenzo phantom.

\section{Discussion and conclusion}

In this work, we have validated a GAMOS simulation of the Raytest ClearPET, a state-of-theart small-animal PET system. The simulation includes the main characteristics of the scanner, namely the use of a dual layer of pixelated crystals with natural radioactivity, the axial shift between detectors, and the rotating gantry. We observed a discrepancy between simulated and measured spatial resolution of $8.4 \%$ (with a maximum FWHM difference over all measurement directions of $0.5 \mathrm{~mm}$ ). Both measured and simulated results show a reduced degradation on the FWHM across the transverse axis (on the order of $1 \mathrm{~mm}$ ), which ClearPET achieves by using two layers of crystals (phoswich configuration) to assess the DOI in the detectors. With regard to the sensitivity and scatter fraction results, they show excellent agreement, with simulated differences of absolute sensitivity less than $1 \%$ for the $250-750 \mathrm{keV}$ energy window. The simulated SF was $31.9 \%$ versus a measured value of $31.0 \%$. Simulated and measured total count rates show agreement better than $5.5 \%$ for a wide range of activities (less than $40 \mathrm{MBq}$ inside a mouse-sized phantom). Although similar results were obtained for the NEC rate at activities below $20 \mathrm{MBq}$, higher discrepancies were found after reaching the peak. We needed to simulate the saturation of the single event transfer to reproduce the behaviour of the system 

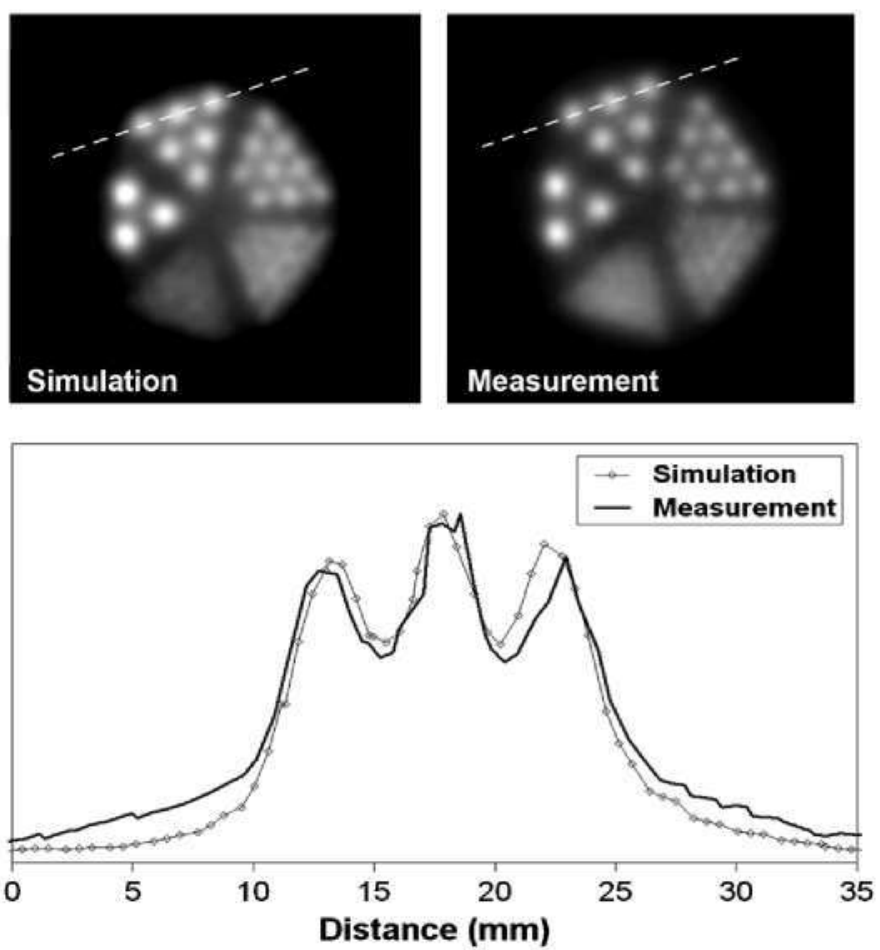

Figure 7. GAMOS simulation and experimental acquisition of a micro Derenzo phantom ( 3 million coincidences). Transaxial view (top), summed over 20 slices, and activity profiles (bottom) along the centres of the $2.5 \mathrm{~mm}$ rods. The dotted lines show the position of the profiles.

when the total coincidences peak is reached. A particular characteristic of the ClearPET is the storage of single events to perform off-line coincidence sorting, which makes the bandwidth on the single transfer (from the cassettes to the preprocessor units) to be the limiting factor on the count rate performance. Therefore, the simulation model provides an appropriate tool to study how different values of the bandwidth can affect the overall performance of the scanner.

The intrinsic radioactivity in the crystals was modelled in order to yield the same number of counts that those measured in the real system. NEMA protocols for systems with natural radioactivity establish the measurement of the coincidences due to the intrinsic radioactivity for a correct assessment of the count rates. The results show how GAMOS can be used to simulate the NEMA counting rate performance for scanners using scintillators containing Lu, such as LYSO, LuYAP, MLS (mixed lutetium silicate) or LSO (lutetium oxyorthosilicate), which is widely present in clinical scanners.

Simulation output data were written in the same format as the ClearPET in order to use the same reconstruction algorithms. As this system uses STIR, an open-source package, this functionality was added to GAMOS and made available for those users interested on PET image reconstruction. The STIR 3D-OSEM algorithm was used to reconstruct a Derenzo-like phantom and we presented the images obtained with simulated and experimental data showing the same quality in both cases.

In conclusion, GAMOS has provided a flexible framework for doing simulations based on GEANT4 physics and particle transportation. The full simulation of the ClearPET required 
the addition of specific features, such as the modelling of its data acquisition process and the generation of output data with equivalent formats. The results show that the GAMOS package is very suitable to simulate PET detectors as well as NEMA experimental setups.

\section{References}

Agostinelli S et al 2003 GEANT4-a sinulation toolkit Nucl. Instrum. Methods. A 506 250-303

Allison $\mathrm{J}$ et al 2006 GEANT4 development and applications IEEE Trans. Nucl. Sci. 53 270-8

Arce P, Rato P, Canadas M and Lagares J I 2008 GAMOS: a GEANT4-based easy and flexible framework for nucleat medicine applications IEEE NSS/MIC 2008 Conf. Record pp 3162-68

Baberdin A, Dutova A. Fedorov A. Korzhik M. Ligoun V. Missevitch O, Kazak V. Vinokurov A and Zagunenov S 2008 (Lu-Y)AlO 3 :Ce scintillator for well loggin IEEE Trans. Nucl. Sct. 55 1170-3

Bao Q, Newport D, Chen M, Stont D B and Chatziioannon A F 2009 Performance evaluation of the inveon dedicated PET preclinical tomograph based on the NEMA NU-4 Standards $J$. Nucl. Med. 50 401-8

Briesmeister J F 2000 MCNP-a general Monte Carlo N-particle transport code version 4C Report no LA-137/19-M (Los Alamos, NM: Los Alamos National Laboratory)

Buvat I, Castiglioni I, Feuardent J and Gilardi M C. 2005 Unified description and validation of Monte Carlo simulators in PET Phys. Med Biol. 50 329-46

Canadas M, Enbid M. Lage E. Desco M, Vaquero J J and Perez J M 2008 Performance comparison of two conmercial small animal PET scannets: ClearPET and rPET-1 IEEE NSS/MIC 2008 Conf. Record pp 4773-9

Castiglioni I, Cremonesi O. Gilardi M C., Bettinardi V, Rizzo G, Savi A, Belloti E and Fazio F 1999 Scatter correction techniques in 3D PET: a Monte Carlo evaluation IEEE Trans. Nucl. Sci. 46 2053-8

Cherry S R and Gambhir S S 2001 Use of positron enission tomography in animal research Inst. Lab. Anim. Res. J. 42 219-32

Daube-Witherspoon M E and Muehllehner G 1987 Treatment of axial data in three-dimensional PET J. Nucl. Med. $281717-24$

España S. Herraiz J L. Vicente E, Vaquero J J. Desco M and Udías J M 2009 PeneloPET, a Monte Carlo PET simulation tool based on PENELOPE: features and validation Phys. Med. Biol 54 1723-42

Fitestone R B and Ekstrom L 1999 Table of tadioactive isotopes LBNL Isotopes Ptoject-LUNDS Universitet. http:/ie.lbl.gov/toit

GAMOS 2010 User`s Guide http:/fismed.ciemat.es/GAMOS/gamos_userguide.php

Hudson H M and Larkin R S 2004 Accelerated image reconstruction using ordered subsets of projection data IEEE Trans. Med. Imaging 13 601-9

Hutchins G D. Miller M A, Soon V C and Receveur T 2008 Snall animal PET imaging Inst. Lab. Amim. Res. J. $4954-65$

Jan S et al 2004 GATE: a simulation toolkit for PET and SPECT Phys. Med. Biol. 49 4543-61

Kuntnet C, Aufftay E. Lecou P, Pizzolotto C and Schneegans M 2002 Intrinsic energy resolution and light output of the $\mathrm{Lu}_{0.7} \mathrm{Y}_{0.3} \mathrm{AP}: \mathrm{Ce}$ scintillator Nucl. Instrum. Methods A 493 131-6

Lewellen T K 2008 Recent developments in PET detector technology Phys. Med. Biol. 53 R287-R317

Lewellen T K, Hartison R L and Vannoy S 1998 The SimSET Program Monte Carlo Calculations in Nuclear Medicine ed M Ljungberg. S Strand and M King (Philadelphia, PA: Institute of Physics Publishing) pp 77-92

Luo W. Anashkin E and Matthews C. G 2010 Performance evaluation of a PEM scanner using the NEMA NU 4-2008 snall animal PET standards IEEE Trans. NuCl. Sci. 57 94-103

Mosset J B et al 2004 Developnent of an optimised LSO/LuYAP phoswich detector head for the ClearPET camera IEEE NSS/MIC 2004 Conf. Record vol 4 pp $2439-43$

Nelson W R, Hitayama H and Rogers D W O 1985 The EGS4 code system Report SLAC-265 Stanford Linear Accelerator Center. Stanford, CA

NEMA 2008 Performance measurements for small animal positron emission tomographs NEMA Standards Publication NU 4-2008, Technical Report (Rosslyn, VA: National Electrical Manufacturers Association)

Pidol L, Kahn-Harari A, Viana B, Virey E, Fertand B, Dotenbos P, De Haas J T M and Van Ejjk C. W E 2004 High efficiency of lutetium silicate scintillators, Ce-Doped LPS, and LYSO crystals IEEE Trans. Nucl. Sci. 51 1084-7

Reilhac A at al 2004 PET-SORTEO: a Monte Callo-based simulator with high count rate capabilities IEEE Trans. Nucl. Sci. $5146-52$

Roldan P S, Canadas M, Dietzel O, Pautror C, Sarasola I and Wagner A 2007 Performance evaluation of Raytest ClearPET. a PET scanner for snall and medium size aninals IEEE NSS/MIC 2007 Conf. Record vol 4 pp $2859-64$ 
Rowland D J and Cherry S R 2008 Small-animal preclinical nuclear medicine instrumentation and methodology Semin. Nucl. Med. $38209-22$

Sempau J et al 1997 An alyorithm for Monte Carlo simulation of the coupled electron-photon transport Nucl. Instrum. Methods B $132377-90$

Streun M et al 2003 Pulse shape discrimination of LSO and LuYAP scintillators for depth of interaction detection in PET IEEE Trans. NuCl. Sci, 50 344-7

Strenn M, Brandenburg G, Larue H, Parl C and Ziemons K 2006 The data acquisition system of ClearPET Neuro-a small animal PET scannet IEEE Trans. Nucl. Sci. $\mathbf{5 3} 700-3$

Strother S C. Casey M E and Hoffman E J 1990 Measuring PET scanner sensitivity: relating countrates to image signal-to-noise ratios using noise equivalent counts IEEE Trans. Nucl. Sici. 37 783-8

Thiel M. Doring W M, Dotmenev V, Dtexlet P, Novotny R W, Rost M and Thomas A 2006 High-energy photon detection with LYSO crystals IEEE Trans. Nucl. Sct. $551425-9$

Thielemans K, Mustafovic S and Tsounpas C 2006 STIR: software for tonographic inage reconstiuction release 2 IEEE NSS/MIC $20 \% 6$ Conf. Record vol 4 pp $2174-6$

Thompson C. J, Cantu J M and Picard Y 1992 PETSIM: Monte Carlo program simulation of all sensitivity and resolution parameters of cylindrical positron imaging systems Phys. Med. Biol. 37 731-49

Yamamoto S, Horii H. Hurutani M. Matsumoto K and Senda M 2005 Investigation of single, random. and true counts from natural radioactivity in LSO-based clinical PET Ann. Nucl. Med. 19 109-14

Yanch J C and Dobrzenjecki A B 1993 Monte Carlo simulation in SPECT: complete 3-D modeling of source, collimator and tomographic data acquisition IEEE Trans. Nucl. Sci. 40 198-203

Weber S and Bauer A 2004 Small animal PET: aspects of performance assessment Eur. J. NuCl. Med. Mol. Imaging 31 1545-55

Wietholt C, Hsiao I, Lin K, Chung Y. Chen C and Yen T 2008 Petformance evaluation of snall animal PET system using NEMA standards J. NuCl. Med. Meeting Abstracts 49119

Zaidi H 1999 Relevance of accurate Monte Catlo modeling in nucleat medical imaging Med. Phys. 26 574-608

Zajdi H, Scheurer A H and Morel C 1999 An object-oriented Monte Carlo simulator for 3D cylindrical positron tomographs Comput. Methods Programs Biomed 58 133-45

Ziemons $\mathrm{K}$ et al 2003 The ClearPET ${ }^{\mathrm{TM}}$ LSO/LuYAP phoswich scanner: a high performance small animal PET system IEEE NSS/MIC 2003 Conf. Record vol 3 pp 1728-32 